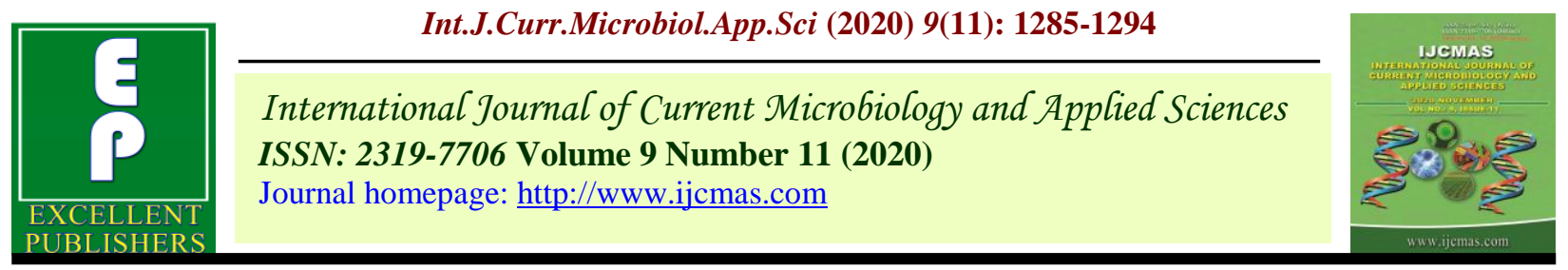

Original Research Article

https://doi.org/10.20546/ijcmas.2020.911.150

\title{
Phytosociological Association of Weeds in Rice Crop of Bundelkhand Region of Uttar Pradesh
}

\author{
Dinesh Sah", G.S. Panwar, A.H. Kalhapure and Narendra Singh \\ Department of Agronomy, College of Agriculture, Banda University of Agriculture and \\ Technology, Banda, Uttar Pradesh, India \\ *Corresponding author
}

\begin{abstract}
A B S T R A C T
Keywords

Bundelkhand, Rice, Weed diversity, weed survey

Article Info

Accepted:

10 October 2020 Available Online: 10 November 2020

The field study was conducted to investigate weed flora diversity in rice crop during kharif season of 2020-21 in four development blocks viz. Naraini, Mahua, Badokhar Khurd and Baberu of Banda District in Uttar Pradesh State. This study identified and quantified the floristic composition of weeds in four different blocks. The number of monocot species recorded in the study was $13(68 \%)$, while the number of dicot species was $6(32 \%)$. Out of 19 weed species 9 were annual, 7 perennial and remaining 3 were annual/perennial. Among several weeds Cyanotis axillaris, Cyperus difformis, Cyperus iria, Cyperus rotundus, Echinochloa colona, Echinochloa crus-galli, Eclipta alba, Fimbristylis miliaceae, Ludvwigia hyssopifolia and Monochoria vaginalis were present at all four study sites. Importance Value Index (IVI) of Monochoria vaginalis was 44.53 and 44.45 at Naraini and Mahua blocks, respectively. While, IVI value of Scirpus muritimus was 85.78 at Badokhar Khurd and IVI value of Caesulia axillaris at Baberu block was 40.96 . Shannon diversity index was highest 3.01 BadokharKurd block, which was followed by Mahua (2.57), Naraini (2.56) and Baberu blocks (2.43). Weed flora at Naraini when compared with site Baberu was found more dissimilar (0.226) than the comparison between Badokhar Khurd and Mahua blocks. Weed species were uniformly distributed in rice field of Badokhar Khurd than other sites as indicated by Evenness Index.
\end{abstract}

\section{Introduction}

Rice (Oryza sativa L.) is the most important staple food crops of the world. More than half of the human population depends on rice for their daily food which ranks third in world for production, after maize and wheat. Beside human food, rice is a source for number of industrial products like rice starch, rice bran oil, flaked rice, puffed rice and rice husk etc. In India, rice is grown in an area of 43.86 million hectare with a production of 104.32 million tonnes and an average productivity of $2.4 \mathrm{t} \mathrm{ha}^{-1}$. Out of the total $43.86 \mathrm{Mha}$ area under rice cultivation, puddled rice culture occupies 56\% (Anonymous, 2016). It is prominently grown during kharif season in Uttar Pradesh. Farmers of Bundelkhand region also cultivating rice on larger area during rainy season. Banda district of UP Bundelkhand is receiving $850-900 \mathrm{~mm}$ annual rainfall mostly during monsoon 
season. Few development blocks of district have assured irrigation facility on which rice is grown as major crop during kharif followed by wheat during rabi season. Rice is cultivated in India in a very wide range of ecosystems from irrigated to shallow lowlands, mid-deep lowlands, and deep water to uplands. Transplanting is the major method of rice cultivation in India. In the rice agroecosystems ideal environment conditions are provided for optimal rice productivity are being exploited by the associated weeds. Weeds are the serious constraints in ricewheat cropping system. Weed infestation is one of the factors responsible for low productivity (Singh et al., 2015) India loses agricultural produce worth over $\$ 11$ billion due to weeds (Anonymous 2018). Economic losses of about $\$ 4.42$ billion in rice has been observed due to weeds, which are followed by wheat (\$3.376 billion) and soybean (\$1.56 billion).

In India, rice is grown under a wide variety of cultural practices in different agro-ecological conditions that is why weed diversity varied (Duary et al., 2015). Dynamic, composition and competition by weeds is dependent on soil, climate, cropping and management factors. Rice fields can be colonized by terrestrial, semi aquatic or aquatic plants depending on the type of rice culture and season. The total number of weeds species in a field largely depends on the associated environment and cropping systems. Moreover, selective control of weeds in crop fields requires the knowledge of the most important weeds associated with a particular crop for effective targeted control. There is scarcity of information on the most important weed species associated with rice and their phytosociological attributes in the study area which resulted to farmers investing extra time and resources in controlling less important weeds in the field.
Phyto-sociological study gives an assessment of plants or weeds of importance in a particular area with fact and figures; provide overall information on the species-wise distribution in and around crops of a given area and; compare and classify weeds in a crop-weed ecosystem (Das, 2008). Understanding the sociological structure of weeds in crop fields is a pre-requisite for its effective management. Phytosociological studies of weeds are necessary for understanding the relationship between crops and their weed flora and may be useful, as a tool for developing a sustainable long-term weed management strategy. Moreover, such studies are helpful in determining how a weed population changes over time in response to selective pressures due to field management practices. This study was under taken to determine the phytosociological characters of weeds in rice field and suggest an effective weed management strategy.

\section{Materials and Methods}

The observation was conducted at four selected development blocks of Banda district of U.P. Bundelkhand viz. Naraini, Mahua, BadokharKhurd and Baberu. These development blocks of district having large area under canal irrigation, and mainly used for paddy cultivation during kharif and wheat during rabi season. The climate of region is typical subtropical with long dry season from late September to end of June and wet season from July to September with hot desiccating winds in summer (May-June) with intensive evapo-transpiration losses.

This field-based survey was carried-out during September month of 2020. At this stage, approximately two months would have gone after weeding. This time chosen for observation because, most of the weeds were well established, most of them were in flowering or seed setting stages. Frequent 
visits were made to the rice crop fields and the specimens collected were identified with the help of available literature.

Weed species compositions in the fields were assessed by throwing $0.25 \mathrm{~m}^{2}$ quadrate randomly in 10 different locations in each development block. The structure and composition of vegetation in the agricultural fields have been compared in terms of frequency, density, abundance and their relative values were derived from the primary data (Curtis 1959).

The method for calculating various phytosociological attributes studied are described as:

Frequency $(\mathrm{F})=$ Number of quadrates in which the species occurs /Number of quadrates studied Relative Frequency $(\mathrm{RF})=$ (Frequency value for a species/Total of Frequency value for all the species) $\times 100$

Density (D) $=$ Total number of individuals of a species in all the quadrates/Number of quadrates studied

Relative Density $(\mathrm{RD})=($ Density value for a species/Total of Density value for all the species) $\times 100$

Abundance $(\mathrm{A})=$ Total number of individuals of a species/Number of quadrates in which the species occurs

Relative abundance $(\mathrm{RA})=($ Abundance value for a species/Total of abundance value for all the species) $\mathrm{x} 100$

\section{Importance Value Index (IVI) (Phillips 1959)}

Important Value Index is valuable statistical measures for the analysis of phytosociology and plant community and it provides an overall idea of a species and its importance in the plant community. It is derived by summing up Relative Frequency, Relative Density and Relative Abundance.

Importance Value Index (IVI) $=\mathrm{RA}+\mathrm{RD}+$ RF

\section{Species Diversity Index (Shannon-Weiner 1963)}

Shannon-Weiner Index (1963) is one of the widely used indices for measuring species diversity. Shannon-wiener index $(\mathrm{H})=-\mathrm{S}[\mathrm{Pi}$ (ln Pi)]

Here $\mathrm{Pi}=($ Number of individual of one weed species/Total number of all individual of weed species) $\times 100$

\section{Evenness index (Pielou 1977)}

Evenness index $(E)=H / H m a x$. or $=H / \log S$

Here $\mathrm{H}=$ Shannon wiener diversity index $\mathrm{S}=$ Total number of species

Species richness: Species richness is another mode of expression of the diversity and based on the total number of species and total number of individuals in a sample or habitat.

Richness Index $\mathrm{D}=\mathrm{S} / \sqrt{\mathrm{N}}$ Where, ' $\mathrm{D}$ ' is the index value ' $S$ ' total number of species ' $N$ ' total number of individuals of all species.

\section{Similarity index (Sorensen's Index)}

Similarity index is a measure of the relative abundance of the different species making up the richness of an area (Azizi 2016).

Similarity index $(S)=2 C /(A+B)$

Here $\mathrm{A}=$ Number of species in one crop, $\mathrm{B}=$ Number of species in another crop, $\mathrm{C}=$ Number of species common in both crop 


\section{Dissimilarity index}

Dissimilarity index $=1-\mathrm{S}$, Here $\mathrm{S}=$ Similarity index

\section{Results and Discussion}

\section{Composition of weed species}

Nineteen weed species belonging to 07 families were found in all the different observation site of rice fields. The type and number of weeds vary in the site studied at different blocks. Maximum number of weed species were present in the site one and two (17), followed by site third (15) and site fourth (14).The floristic composition of recorded weed species was grouped into Monocotyledons and Dicotyledons.

The number of monocot species recorded in the study was $13(68 \%)$, while the number of dicot species was $6(32 \%)$. Among several categories of weeds, broad leaved weed (40\%) were pre dominant in all four observation sites. In a study Manisankar et al., (2020) also observed 39.5 per cent density of broad leaf weeds in transplanted rice. Out of 19 weed species 9 were annual, 7 were perennial and remaining 3 were annual/perennial weeds. Family Poaceae were represented by 6 species, Cyperaceae by 5 species, Asteraceae by 3 species, Commelinaceae by 2 species; the other remaining 3 families were represented by 1 species each (Table 1).

Among several weeds Cyanotis axillaris, Cyperus difformis L., Cyperus iria L., Cyperus rotundus L., Echinochloa colona (L.) Link, Echinochloa crus-galli L., Eclipta alba (L.) Hassk, Fimbristylis miliaceae (L) Vahl, Ludvwigia hyssopifolia and Monochoria vaginalis were present in all four study sites. Out of 19 weeds, 10 were available at all 4 locations, 6 at three locations and 2 at two locations only. Weed Caesulia axillaris was found only in one study site (Table 1).

\section{Frequency, density and abundance}

The frequency, density and abundance of various weed species under the prevailing environmental set up presented in Table 2. In Naraini block, highest frequency (0.8) of weed population was recorded for Monochoria vaginalis, followed by 0.5 for Ludvwigia hyssopifolia while 0.4 for Paspalum disticum. In Mahua block Monochoria vaginalis occurred with 0.7 frequency while Cyperus iria showed frequency of 0.5. Weed Bracharia reptans, Cynodon dactylon, Eclipta alba and Scirpus maritimus exhibits lower frequency of 0.1 only. In Barokharkhurd block, highest frequency of 0.7 was observed for Cyperus difformis, while Scirpus maritimus had 0.5 frequency. Minimum frequency of 0.1 was associated with Cyanotis axillaris.Under Baberu block maximum frequency value 0.4 was associated with Fimbristylis miliaceae while minimum value 0.1 with Echinochloa colona, Cyperus rotundus and Monochoria vaginalis.

Weed species Monochoria vaginalis showed highest density (1.5) in Naraini block and Mahua block which was followed by Scirpus maritimus (1.2 and 0.7) under these blocks. Highest density of 5.10 was associated with weed Scirpus maritimus in Barokharkhurd block while in Baberu block Fimbristylis miliaceae exert maximum density of 0.90 . Most of the weed species reflecting lower density values indicating single plant dominated community structure of the weed flora.

In Naraini block weed abundance value ranges between 1-6, in Mahua block between 0.50 -7, in Barokharkhurd block 1-10.20 and in Baberu block 1-4.5. The weeds with 
maximum abundance in Naraini, Mahua and Barokharkhurd block was Scirpus maritimus with abundance value 6,7 and 10.20, respectively while in Baberu block Caesulia axillaris with 4.5 abundance value. Eight weeds of Naraini block and four weeds of
Barokharkhurd and Baberu block showed least abundance value of 1.0, Fimbristylis miliaceae exert least abundance value 0.50 in Mahua block. This is likely to be as a result of difference in cultural and weed management practices.

Table.1 Floristic composition of the weed flora in the rice fields

\begin{tabular}{|c|c|c|c|c|c|}
\hline S no & $\begin{array}{l}\text { Botanical Name of } \\
\text { Weed species }\end{array}$ & $\begin{array}{l}\text { Common name of } \\
\text { weed species }\end{array}$ & Family & Group & Life cycle \\
\hline 1. & $\begin{array}{l}\text { Brachiaria reptans } \\
\text { (L) Gardner \& CE }\end{array}$ & $\begin{array}{l}\text { Leman ghas, Para } \\
\text { ghas }\end{array}$ & Poaceae & Monocot & $\begin{array}{l}\text { Annual// } \\
\text { Perennial }\end{array}$ \\
\hline 2. & Caesulia axillaris & Ganthila, Galfule & Asteraceae & Dicot & Annual \\
\hline 3. & $\begin{array}{l}\text { Commelina diffusa } \\
\text { Burn F. }\end{array}$ & Ken, Kenna & Commelinaceae & Monocot & Annual \\
\hline 4. & Cyanotis axillaris & Garenda, Chara & Commelinaceae & Monocot & Perennial \\
\hline 5. & Cynodon dactylon $\mathrm{L}$. & Doobghas & Poaceae & Monocot & Perennial \\
\hline 6. & Cyperus difformis L. & $\begin{array}{l}\text { Dilla, } \\
\text { Ganthvalamotha, } \\
\text { Gond }\end{array}$ & Cyperaceae & Monocot & Perennial \\
\hline 7. & Cyperus iria $\mathrm{L}$. & Nagar motha, Gond & Cyperaceae & Monocot & Perennial \\
\hline 8. & Cyperus rotundus $\mathrm{L}$. & Motha, Gond & Cyperaceae & Monocot & Perennial \\
\hline 9. & $\begin{array}{l}\text { Echinochloa colona } \\
\text { (L.) Link }\end{array}$ & $\begin{array}{l}\text { Jangal rice, } \\
\text { Jangalghas, Sawan }\end{array}$ & Poaceae & Monocot & Annual \\
\hline 10 & $\begin{array}{l}\text { Echinochloa crus- } \\
\text { galli L. }\end{array}$ & Sawak, sanwan & Poaceae & Monocot & Annual \\
\hline 11 & $\begin{array}{l}\text { Eclipta alba (L.) } \\
\text { Hassk }\end{array}$ & Bhangara, Bhringraj & Asteraceae & Dicot & Annual \\
\hline 12 & $\begin{array}{l}\text { Enhydra fluctuans } \\
\text { Lour }\end{array}$ & Jalbrahmi, Helkuch & Asteraceae & Dicot & Annual \\
\hline 13 & $\begin{array}{l}\text { Eragrostis pilosa } \\
\text { tenella (L.) Beauv. }\end{array}$ & Lavgrass, mutmur & Poaceae & Monocot & Annual \\
\hline 14 & $\begin{array}{l}\text { Fimbristylis } \\
\text { miliaceae (L) Vahl }\end{array}$ & Jhiruva & Cyperaceae & Monocot & Perennial \\
\hline 15 & $\begin{array}{l}\text { Ipomoea aquatic } \\
\text { Forsk }\end{array}$ & $\begin{array}{l}\text { Jaliypalak, Karmu, } \\
\text { Karmi }\end{array}$ & Convolvulaceae & Dicot & $\begin{array}{l}\text { Annual/ } \\
\text { Perennial }\end{array}$ \\
\hline 16 & $\begin{array}{l}\text { Ludvwigia } \\
\text { hyssopifolia }\end{array}$ & Ptua, Pile fulvalibuti & Onagraceae & Dicot & Annual \\
\hline 17 & $\begin{array}{l}\text { Monochoria } \\
\text { vaginalis }\end{array}$ & Pond weed, Nanka & Pontederiaceae & Dicot & $\begin{array}{l}\text { Annual } \\
\text { /Perennial }\end{array}$ \\
\hline 18 & Paspalum disticum & Kodo, hunka & Poaceae & Monocot & Annual \\
\hline 19 & Scirpus maritimus L & Buchad, Dilli & Cyperaceae & Monocot & Perennial \\
\hline
\end{tabular}


Table.2 The frequency, density and abundance of different weed species in rice crop at the observation site

\begin{tabular}{|c|c|c|c|c|c|c|c|c|c|c|c|c|}
\hline \multirow[t]{2}{*}{ Weed species } & \multicolumn{3}{|c|}{ Naraini Block } & \multicolumn{3}{|c|}{ Mahua Block } & \multicolumn{3}{|c|}{$\begin{array}{c}\text { Badokharkhurd } \\
\text { Block }\end{array}$} & \multicolumn{3}{|c|}{ Baberu Block } \\
\hline & $\mathbf{F}$ & D & $\mathbf{A}$ & $\mathbf{F}$ & D & $\mathbf{A}$ & $\mathbf{F}$ & D & $\mathbf{A}$ & $\mathbf{F}$ & D & $\mathbf{A}$ \\
\hline $\begin{array}{l}\text { Brachiaria reptans }(\mathrm{L}) \\
\text { Gardner \& CE }\end{array}$ & 0.2 & 0.20 & 1.00 & 0.1 & 0.20 & 2.00 & 0.40 & 0.40 & 1.00 & 0.0 & 0.0 & 0.0 \\
\hline Caesulia axillaris & 00 & 00 & 00 & 0 & , & 0 & 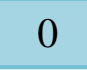 & 0 & 0 & .2 & 0.90 & 4.50 \\
\hline Commelina diffusa Burn F. & 0.3 & 0.30 & 1.00 & 0 & 0.00 & 0.00 & 0.4 & 1.10 & 0.00 & 0.2 & 0.50 & 0.00 \\
\hline Cyanotis axillaris & 0.2 & 0.20 & 1.00 & 0.2 & 0.30 & 1.50 & 0.1 & 0.10 & 1.00 & 0.3 & 0.30 & 1.00 \\
\hline Cynodon dactylon $\mathrm{L}$. & 0 & 0.00 & 0.00 & 0.1 & 0.10 & 1.00 & 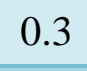 & 0.40 & 1.33 & 0.2 & 0.30 & 1.50 \\
\hline Cyperus difformis $\mathbf{L}$. & 0.3 & 0.40 & 1.33 & 0.3 & 0.40 & 1.33 & 0.1 & 2.00 & 2.86 & 0.2 & 0.70 & 3.50 \\
\hline Cyperus iria $\mathrm{L}$. & 0.3 & 0.40 & 1.33 & 0.5 & 0.80 & 1.60 & 0.2 & 0.40 & 2.00 & 0.3 & 0.50 & 1.67 \\
\hline Cyperus rotundus $\mathbf{L}$. & 0.1 & 0.10 & 1.00 & 0.2 & 0.20 & 1.00 & 0.2 & 0.20 & 1.00 & 0.1 & 0.10 & 1.00 \\
\hline $\begin{array}{l}\text { Echinochloa colona (L.) } \\
\text { Link }\end{array}$ & 0.3 & 0.40 & 1.33 & 0.3 & 0.40 & 1.33 & 0.3 & 0.40 & 1.33 & 0.1 & 0.10 & 1.00 \\
\hline Echinochloa crus-galli L. & 0.3 & & 2.00 & 0.3 & & & & 0 & & 0.4 & 0.80 & 4.00 \\
\hline Eclipta alba (L.) Hassk & 0 & & 1 & 0 & & & & 0 & & 0 & 0.40 & 0.00 \\
\hline Enhydra fluctuans Lour & 0 & & 1 & 0 & & & 0 & & & 0 & 0.00 & 0.00 \\
\hline $\begin{array}{l}\text { Eragrostis pilosa tenella (L.) } \\
\text { Beauv. }\end{array}$ & 0.1 & 0.10 & 1.00 & 0.3 & 0.30 & 1.00 & 0 & 0.00 & 0.00 & 0 & 0 & 0 \\
\hline $\begin{array}{l}\text { Fimbristylis miliaceae (L) } \\
\text { Vahl }\end{array}$ & 0.2 & 0.20 & 1.00 & 0.2 & 0.10 & 0.50 & 0.2 & 0.20 & 1.00 & 0.4 & 0.90 & 2.25 \\
\hline Ipomoea aquatic Forsk & 0.2 & 0.20 & 1.00 & 0.2 & 0.20 & 1.00 & 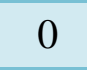 & 0.00 & 0.00 & 0.2 & 0.20 & 0.00 \\
\hline Ludvwigia hyssopifolia & 0.5 & 0.50 & 1.00 & 0.3 & 0.30 & 1.00 & 0.4 & 0.50 & 1.25 & 0.3 & 0.40 & 1.33 \\
\hline Monochoria vaginalis & 0.8 & 1.50 & 1.88 & 0.7 & 1.50 & 2.14 & 0.4 & 0.50 & 1.25 & 0.1 & 0.10 & 1.00 \\
\hline Paspalum disticum & 0.4 & 0.50 & 1.25 & 0.3 & 0.50 & 1.67 & 0.4 & 0.50 & 1.25 & 0 & 0.00 & 0.00 \\
\hline Scirpus maritimus $\mathbf{L}$ & 0.2 & 1.20 & 6.00 & 0.1 & 0.70 & 7.00 & 0.5 & 5.10 & 10.20 & 0 & 0.00 & 0.00 \\
\hline
\end{tabular}

$\mathrm{F}=$ Frequency, $\mathrm{D}=$ Density, $\mathrm{A}=$ Abundance 
Table.3 The relative frequency, relative density, relative abundance and IVI of different weed species at the observation site

\begin{tabular}{|c|c|c|c|c|c|c|c|c|c|c|c|c|c|c|c|c|}
\hline \multirow[t]{2}{*}{ Weed species } & \multicolumn{4}{|c|}{ Naraini Block } & \multicolumn{4}{|c|}{ Mahua Block } & \multicolumn{4}{|c|}{ Barokharkhurd Block } & \multicolumn{4}{|c|}{ Baberu Block } \\
\hline & RF & RD & RA & IVI & RF & RD & RA & IVI & RF & RD & RA & IVI & RF & RD & RA & IVI \\
\hline $\begin{array}{l}\text { Brachiaria reptans }(\mathrm{L}) \\
\text { Gardner \& CE }\end{array}$ & 4.17 & 2.74 & 3.90 & 10.81 & 2.27 & 2.82 & 6.92 & 12.01 & 7.84 & 3.15 & 3.51 & 14.50 & 0.00 & 0.00 & 0.00 & 0.00 \\
\hline Caesulia axillaris & 0 & 0 & 0 & 00 & 0 & 0 & 0 & 0 & 0 & 0 & 0 & 0 & 6.67 & 14.52 & 19.78 & 40.96 \\
\hline $\begin{array}{l}\text { Commelina diffusa Burn } \\
\text { F. }\end{array}$ & 6.25 & 4.11 & 3.90 & 14.26 & 0.00 & 0.00 & 0.00 & 0.00 & 7.84 & 8.66 & 0.00 & 16.50 & 6.67 & 8.06 & 0.00 & 14.73 \\
\hline Cyanotis axillaris & 4.17 & 2.74 & 3.90 & 10.81 & 4.55 & 4.23 & 5.19 & 13.96 & 1.96 & 0.79 & 3.51 & 6.26 & 10.00 & 4.84 & 4.40 & 19.23 \\
\hline Cynodon dactylon $\mathrm{L}$. & 0.00 & 0.00 & 0.00 & 0.00 & 2.27 & 1.41 & 3.46 & 7.14 & 5.88 & 3.15 & 4.68 & 13.71 & 6.67 & 4.84 & 6.59 & 18.10 \\
\hline Cyperus difformis $\mathbf{L}$. & 6.25 & 5.48 & 5.20 & 16.93 & 6.82 & 5.63 & 4.61 & 17.06 & 13.73 & 15.75 & 10.03 & 39.51 & 6.67 & 11.29 & 15.38 & 33.34 \\
\hline Cyperus iria $\mathrm{L}$. & 6.25 & 5.48 & 5.20 & 16.93 & 11.36 & 11.27 & 5.53 & 28.17 & 3.92 & 3.15 & 7.02 & 14.10 & 10.00 & 8.06 & 7.33 & 25.39 \\
\hline Cyperus rotundus L. & 2.08 & 1.37 & 3.90 & 7.36 & 4.55 & 2.82 & 3.46 & 10.82 & 3.92 & 1.57 & 3.51 & 9.01 & 3.33 & 1.61 & 4.40 & 9.34 \\
\hline $\begin{array}{l}\text { Echinochloa colona (L.) } \\
\text { Link }\end{array}$ & 6.25 & 5.48 & 5.20 & 16.93 & 6.82 & 5.63 & 4.61 & 17.06 & 5.88 & 3.15 & 4.68 & 13.71 & 3.33 & 1.61 & 4.40 & 9.34 \\
\hline $\begin{array}{l}\text { Echinochloa crus-galli } \\
\text { L. }\end{array}$ & 6.25 & 8.22 & 7.80 & 22.27 & 6.82 & 9.86 & 8.07 & 24.75 & 5.88 & 3.15 & 4.68 & 13.71 & 6.67 & 12.90 & 17.58 & 37.15 \\
\hline Eclipta alba (L.) Hassk & 4.17 & 4.11 & 5.85 & 14.13 & 2.27 & 1.41 & 3.46 & 7.14 & 5.88 & 3.94 & 5.85 & 15.67 & 6.67 & 6.45 & 0.00 & 13.12 \\
\hline Enhydra fluctuans Lour & 4.17 & 2.74 & 3.90 & 10.81 & 4.55 & 4.23 & 5.19 & 13.96 & 0.00 & 0.00 & 0.00 & 0.00 & 0.00 & 0.00 & 0.00 & 0.00 \\
\hline $\begin{array}{l}\text { Eragrostis pilosa tenella } \\
\text { (L.) Beauv. }\end{array}$ & 2.08 & 1.37 & 3.90 & 7.36 & 6.82 & 4.23 & 3.46 & 14.50 & 0.00 & 0.00 & 0.00 & 0.00 & 0 & 0 & 0 & 0 \\
\hline $\begin{array}{l}\text { Fimbristylis miliaceae } \\
\text { (L) Vahl }\end{array}$ & 4.17 & 2.74 & 3.90 & 10.81 & 4.55 & 1.41 & 1.73 & 7.68 & 3.92 & 1.57 & 3.51 & 9.01 & 13.33 & 14.52 & 9.89 & 37.74 \\
\hline Ipomoea aquatic Forsk & 4.17 & 2.74 & 3.90 & 10.81 & 4.55 & 2.82 & 3.46 & 10.82 & 0.00 & 0.00 & 0.00 & 0.00 & 6.67 & 3.23 & 0.00 & 9.89 \\
\hline Ludvwigia hyssopifolia & 10.42 & 6.85 & 3.90 & 21.17 & 6.82 & 4.23 & 3.46 & 14.50 & 7.84 & 3.94 & 4.39 & 16.17 & 10.00 & 6.45 & 5.86 & 22.31 \\
\hline Monochoria vaginalis & 16.67 & 20.55 & 7.32 & 44.53 & 15.91 & 21.13 & 7.41 & 44.45 & 7.84 & 3.94 & 4.39 & 16.17 & 3.33 & 1.61 & 4.40 & 9.34 \\
\hline Paspalum disticum & 8.33 & 6.85 & 4.88 & 20.06 & 6.82 & 7.04 & 5.77 & 19.63 & 7.84 & 3.94 & 4.39 & 16.17 & 0.00 & 0.00 & 0.00 & 0.00 \\
\hline Scirpus maritimus $\mathbf{L}$ & 4.17 & 16.44 & 23.41 & 44.02 & 2.27 & 9.86 & 24.21 & 36.35 & 9.80 & 40.16 & 35.82 & 85.78 & 0.00 & 0.00 & 0.00 & 0.00 \\
\hline
\end{tabular}

$\mathrm{RF}=$ Relative Frequency, $\mathrm{RD}=$ Relative Density, RA= Relative Abundance, IVI= Importance Value Index 
Table.4 H Index, Evenness Index and Richness Index

\begin{tabular}{|l|c|c|c|c|}
\hline Observation site\Indices & $\begin{array}{c}\text { Naraini } \\
\text { Block }\end{array}$ & Mahua Block & $\begin{array}{c}\text { Barokharkhurd } \\
\text { Block }\end{array}$ & Baberu Block \\
\hline Shannon Diversity Index (H) & 2.56 & 2.57 & 3.01 & 2.43 \\
\hline Shannon Evenness Index (E) & 0.90 & 0.91 & 1.11 & 0.92 \\
\hline Richness Index & 1.99 & 2.02 & 1.33 & 1.78 \\
\hline
\end{tabular}

Table.5 Similarity Index

\begin{tabular}{|l|c|c|c|c|}
\hline \multicolumn{1}{|c|}{ Crops } & $\begin{array}{c}\text { Naraini } \\
\text { Block }\end{array}$ & Mahua Block & $\begin{array}{c}\text { Barokharkhurd } \\
\text { Block }\end{array}$ & Baberu Block \\
\hline Naraini Block & ----- & 0.882 & 0.875 & 0.774 \\
\hline Mahua Block & & ---- & 0.875 & 0.774 \\
\hline Barokharkhurd Block & & & --- & 0.827 \\
\hline Baberu Block & & & & --- \\
\hline
\end{tabular}

Table.6 Dissimilarity Index

\begin{tabular}{|l|c|c|c|c|}
\hline \multicolumn{1}{|c|}{ Crops } & Naraini Block & Mahua Block & $\begin{array}{c}\text { Barokharkhurd } \\
\text { Block }\end{array}$ & Baberu Block \\
\hline Naraini Block & ----- & 0.118 & 0.125 & 0.226 \\
\hline Mahua Block & & ---- & 0.125 & 0.226 \\
\hline $\begin{array}{l}\text { Barokharkhurd } \\
\text { Block }\end{array}$ & & ---- & 0.173 \\
\hline Baberu Block & - & & & ----- \\
\hline
\end{tabular}

Relative values of frequency, density, abundance and importance value index

Values represented in Table 3 reflect considerable variation among the different observed weed species. The lower relative frequency values represent less occurrence and higher frequency values represent more occurrence of weed species. In Naraini block, highest relative frequency was noticed with Monochoria vaginalis (16.67), which was closely followed by Ludvwigia hyssopifolia (10.42) and Paspalum disticum (8.33), and minimum (2.08) with Cyperus rotundus and Eragrostis pilosa. Maximum relative density (20.55) and IVI value 44.53 found with Monochoria vaginalis was most dominant among the observed weed community.
Highest relative abundance value of 23.41, 24.21 and 40.15 was associated with weed species Scirpus maritimus at three locations of study site viz. Naraini, Mahua and Barokharhkurd, respectively.

In Mahua block, relative frequency value varies from 2.27- 15.91, while relative density from 1.41- 21.3. Weed Monochoria vaginalis showed maximum value of relative frequency (15.91), relative density (88.421.135) and IVI value 44.45. Weed species Scirpus maritimus (IVI 36.35) and Cyperus iria (IVI 28.17) also observed as important among sedge weeds, while Echinochloa crus-galli with IVI value 24.75 among grassy weeds. Sridevi et al., (2013) reported Echinochloa crus-gallias predominant weed among grasses and 
Cyperus difformis and Fimbristylis miliacea among sedge weed.

Relative frequency (9.80), relative density (40.16), relative abundance (35.82) and IVI value (85.78) of Scirpus maritimus was recorded in Barokharkhurd block. Thus, Scirpus maritimus is the dominant weed species of the rice crop in concerned study area. In rice field of Baberu block weed Fimbristylis miliaceae showed higher relative frequency (13.33), while higher value of relative density (14.52) was associated with Caesulia axillaris in rice crop of concerned study area. Highest relative abundance value 19.78 and IVI value 40.96 was noticed with weed Caesulia axillari. Observations described above clearly indicate that Monochoria vaginalis and Scirpus maritimus and Caesulia axillaris have been found to be most frequently distributed and important weed species. Singh et al., (2014) at Varanasi and Ramchandra et al., (2010) at Banglore also found Scirpus maritimus and Caesulia axillaris as major weed of rice crop.

\section{Diversity indices}

Shannon's H Index of weed flora diversity was found higher in rice field of Barokharkhurd block (3.01) followed by 2.57 and 2.56 in Mahua and Naraini block, respectively, which was recorded lower in rice field of Baberu block (2.43). Rice field of Barokharkhurd block showed the highest weed diversity with Shannon index $(\mathrm{H}>1.0)$. Similarly, Shannon Evenness Index (E) was highest (1.11) in Barokharkhurd block followed by in Baberu block (0.92). Weed Richness Index of Mahua block was 2.02, higher than all other observation site (Table 4). The highest evenness index means weed species were uniformly distributed in it. Whereas, the lowest evenness index indicates the species were clustered within their habitat and therefore not evenly spaced.
The similarity index showed the pattern of similarity between crops/ sites/ treatments. Naraini block show a high similarity index (0.882) of weed flora with Mahua block and (0.875 and 774) with Barokharkhurd and Baberu blocks (Table 5). Weed flora in Naraini block and Mahua block when compared with Baberu block found more dissimilar (0.226) than between Barokharkhurd block (Table 6). Difference in canopy structure as well as cultural practices could be the reason of this diversity, similarity and dissimilarity.

It was concluded that the land use such as cultivation practices, use of inputs, source of irrigation, selection of crops and cropping systems, weed management practices and other cultural practices affects the weed flora composition. The presence of some weeds in three of four observation site indicates their wider adoptability while restriction of some weeds to particular observation site shows their requirement for special condition in order to grow. This survey will provide a base for future weed surveys. However, extensive field studies would be necessary to quantify the abundance and diversity of weeds under various crops and cropping systems of Bundelkhand region.

\section{Acknowledgement}

Authors express their gratitude to the Directorate of Research, Banda University of Agriculture \& Technology, Banda for providing necessary support and facility during the course of study.

\section{References}

Anonymous 2018. India loses farm produce worth $\$ 11 b$ to weeds every year: ICAR. The Hindu Business Line.

Anonymous, 2016. Area and production of cereals. National Food Security 
Mission.

https://www.nfsm.gov.in/StatusPaper/Ri ce 2016.pdf.

Azizi E. 2016. Evaluation of Weed Species Richness and Similarity of Iran's Provinces in the Fields of Some Species of Poaceae Family. International Journal of Life Science and Engineering. 2(2):8-20.

Curtis JT. 1959. The vegetation of Wisconsin; an ordination of plant community. University of Winconsin press, Madison.

Das TK. 2008. Weed Science. Basics and Application. New Delhi. Jain brothers. $901 \mathrm{p}$.

Duary B, Mishra MM, Dash R, Teja KC. 2015. Weed management in lowland rice. Indian Journal of Weed Science. 47:224-232.

Manisankar, G., T. Ramesh and Rathika, S. 2020. Weed Management in Transplanted Rice through Pre Plant Application of Herbicides: A Review. Int.J.Curr.Microbiol.App.Sci. $\quad$ 9(05): 684-692

Philips EA. 1959. Methods of vegetation study. Henry Holt and company, New York.

Pielou EC. 1977. Mathematical Ecology. A Wiley interscience publication, New
York.Pp.364-75.

Ramchandra, C., Denesh, G.R. and Sydanwarulla. 2010. Weed management practices in transplanted rice by using glyphosate. Biennial Conference on "Recent Advances in Weed Science Research-2010”, February 25-26, 2010, Indira Gandhi Krishi Vishwavidyalaya, Raipur (Chhatisgarh). pp. 68.

Shannon CE and Wiener W. 1963.The mathematical theory of communication. University of Juionis Press, Urbana.117. Singh P, Singh P, Singh L, Qayoom S, Lone BA, Kanth RH. 2015. Phytosociological Association of Weeds in Summer-Kharif Crops of Kashmir Valley Under Different Eco-Situations. Journal of Agri Search. 2:183-188.

Singh, A.K., Singh, M.K., Prasad, S.K. and Sakarwar, P. 2014. Sequential herbicide application and nitrogen rates effect on weeds in direct seeded rice (Oryza sativa L.). The Ecoscan 8(3\&4):249252.

Sridevi V., Jeyaraman S., Chinnusamy, C. and Chellamuthu, V. 2013. Weed management in low land rice (Oryza Sativa.) Ecosystem- A Review. International Journal of Agricultural Science and Research. 3 (3):13-22.

\section{How to cite this article:}

Dinesh Sah, G.S. Panwar, A.H. Kalhapure and Narendra Singh. 2020. Phytosociological Association of Weeds in Rice Crop of Bundelkhand Region of Uttar Pradesh. Int.J.Curr.Microbiol.App.Sci. 9(11): 1285-1294. doi: https://doi.org/10.20546/ijcmas.2020.911.150 